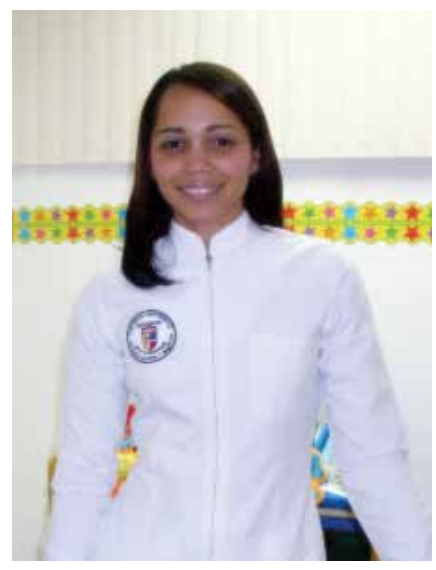

ECOS DESDE LAS FACULTADES

\title{
La enseñanza-aprendizaje en las prácticas de Estomatología
}

\author{
The teaching-learning process in the Stomatology practicum
}

Karina Gutiérrez

Resumen: Se describe el proceso de enseñanza-aprendizaje en las prácticas de la carrera de Estomatología de la Pontificia Universidad Católica Madre y Maestra. Se parte de los 4 principios con los cuales está concebido el plan de estudios, según los requerimientos actuales para la formación del profesional de Estomatología. En este artículo se hace énfasis en la importancia del seguimiento y la evaluación continua del estudiante como garantía de la calidad de la formación profesional.

Abstract: This article describes the teaching-learning process in the practicum for the Bachelor's Degree in Stomatology at the Pontificia Universidad Católica Madre y Maestra. It is based on the four principles that structure the syllabus, according to the current requirements for the education of the Stomatology practitioner. The author stresses the importance of the follow-up and the ongoing evaluation of the student, as the key to ascertain the quality of the professional education.

La carrera de Estomatología de la Pontificia Universidad Católica Madre y Maestra (PUCMM) se fundamenta en cuatro principios básicos: el aprendizaje en servicio, el aprendizaje en condiciones reales, la subordinación del aprendizaje teórico al aprendizaje práctico y el aprendizaje siguiendo un orden de complejidad creciente, considerando que las necesidades odontológicas básicas son las mismas en todas las comunidades humanas [sitio web] www.pucmm.edu.do.

El proceso de enseñanza-aprendizaje en la carrera de Estomatología de la PUCMM se desarrolla con una estrecha relación entre la institución formadora, la comunidad y los servicios. Aprender haciendo es el signo que caracteriza la carrera, pues no se puede comparar hacer las prácticas iniciales en maniquíes, de manera técnica, y esperar a la etapa final de la carrera para hacerlas en pacientes, ya que es con la práctica en situaciones reales que se desarrollan las destrezas.

El proceso de las prácticas juega un rol fundamental en la carrera de Estomatología, quedando demostrado en el cambio de paradigma por el que ha pasado la carrera de Estomatología a través de los años. En este sentido, Salazar (2007) señala que la enseñanza odontológica en América Latina se caracterizó desde sus inicios por un modelo de instrucción individualista, de aspecto puramente biológico, curativo y elitista, sustentado en lo estrictamente teórico y donde el alumno precariamente tenía la oportunidad de aplicar en la práctica los conocimientos adquiridos. En contraste, Suárez (2004) considera que el estomatólogo general debe tener arraigado que esta disciplina se ocupa del hombre como ser bio-psico-social, internamente vinculado a su familia y a su comunidad $y$, por tanto, estos profesionales se deben dedicarse a la promoción, la prevención, la curación y la rehabilitación de la salud bucal.

El diseño curricular de la carrera de Estomatología de la PUCMM se basa en la concepción del plan de estudios como una integración de los contenidos de las disciplinas básicas, pre-clínicas y clínicas desde los primeros años de la carrera, el aprendizaje en servicio como forma organizativa principal del proceso y como vía para que la práctica alcance su verdadera dimensión académica.

\section{Key Words}

Higher Education, dental clinics, practicum, experiential learning, service-learning

* Doctora en Estomatología por la Pontificia Universidad Católica Madre y Maestra, Campus de Santiago. Especialista en Odontopediatría por la Universidad del Salvador, Buenos Aires, Argentina. Profesora por asignatura del Departamento de Estomatología. Para contactar a la autora: kpgutierrezm@gmail.com

Encuentre el texto en "Estrategias de enseñanza-aprendizaje" http://www.pucmm.edu.do/STI/campus/CDP/ComunicaciónPublicaciones/Paginas/CuadernodePedagogiaUniversitaria.aspx

Cuaderno de Pedagogía Universitaria Año 8/ N. 15 /enero - junio 2011 / Santiago, Rep. Dom.: PUCMM / pp.33-35 
Este proceso, además de desarrollarse por niveles, tiene un orden de lo simple a lo complejo, partiendo de un aprendizaje de contenidos y conceptos teóricos, observación del proceso mediante una práctica en vivo o video, práctica en modelos o entre los mismos estudiantes, para luego ir a la atención de pacientes.

La carrera se desarrolla de acuerdo a un sistema modular: en cada semestre del plan de estudios se presentan componentes teóricos, teórico-prácticos y prácticos Los módulos teóricos incluyen los contenidos actualizados que permiten al estudiante alcanzar los objetivos necesarios en cuanto a conceptos fundamentales que deben manejar para realizar su práctica; los teórico-prácticos son aquellos en los cuales el componente teórico se complementa con prácticas en dientes extraídos o modelos para afianzar los conceptos teóricos antes de hacer estas actividades en los pacientes. En algunos casos, estas prácticas son desarrolladas entre los mismos estudiantes, como es el caso de la colocación de anestésicos.

Las prácticas con pacientes se realizan en los tres niveles de atención que constituyen la Carrera de Estomatología: Atención Primaria, Atención Básica y Atención Integral.

Desde el nivel Atención Primaria, que se cursa en el segundo año de universidad y primer año de carrera, se hace énfasis en ver y valorar a cada paciente como un ser bio-sico-social, por lo que no solo se evalúa la condición estomatológica que presenta, sino que se indaga en los aspectos que conforman un ser humano en su totalidad y dándole importancia a los mismos; es decir, el profesorado se enfoca en hacer que los estudiantes comprendan que en el sillón dental está un paciente con necesidades y no en ver simplemente si el paciente necesita una limpieza o un extracción, por ejemplo. Se ofrecen las atenciones odontológicas primarias gratuitas, en clubes, escuelas y zonas de escasos recursos donde las personas acuden en busca de atención. En este nivel, los estudiantes realizan las actividades bajo la supervisión de dos docentes asignados, pues son grupos de 25 a 30 estudiantes. Los docentes dan un seguimiento paso a paso a cada proceso, desde el diagnóstico hasta el resultado logrado. La distribución de estos docentes puede darse ya sea cada uno tomando un grupo de estudiantes, o de acuerdo a las actividades que realicen los mismos, intercambiando día tras día, lo que permite que todos los estudiantes tengan contacto con ambos profesores.

Actualmente el Nivel Atención primaria está ofreciendo servicios en el Children International de Cien Fuegos y en la Iglesia Católica de Guayabal, tanto en la tanda matutina como en la vespertina, llegando a atender un promedio de 40 pacientes por tanda. Este es un aporte de la PUCMM a la comunidad, con lo cual la población recibe el beneficio de la atención odontológica y nuestros estudiantes desarrollan sus prácticas iniciales en condiciones reales. Los estudiantes se distribuyen en parejas de trabajo, lo que permite hacer la función alterna de operador-auxiliar.

Los niveles Atención Básica y Atención Integral se desarrollan en las diferentes clínicas que dispone la PUCMM, tanto en el Campus de
Santiago, (edificio de Ciencias de Salud, tres tandas de atención diaria), como en las clínicas periféricas, en el Hospital Regional Universitario José María Cabral y Báez, Hospital Arturo Grullón, Centro de Atención Primaria Juan XXIII, Hospital Dr. Rafael Castro en Cienfuegos, Centro Periférico Ensanche Libertad, Clínica Estomatológica de Licey, Clínica Estomatológica de Gurabo y Clínica Estomatológica Parque Industrial Zona Franca. Los pacientes acuden a estas clínicas en busca de servicios odontológicos recibiendo servicios de calidad a muy bajo costo.

El nivel Atención Básica incluye las actividades destinadas a resolver los problemas de mayor prevalencia en salud bucal en infantes y adultos; al contar con equipos especializados se realizan actividades más complejas. En este nivel los estudiantes disponen del docente encargado de la clínica, así como de docentes especialistas de diferentes áreas que asisten determinado día a la semana para hacer prácticas interdisciplinarias y, así, facilitar al paciente todos los servicios en un mismo lugar. Se mantiene la supervisión del docente sobre cada actividad, desde el diagnóstico hasta la elaboración del plan de tratamiento en el cual se organizan las actividades que se realizarán al paciente con la secuencia determinada.

El nivel Atención Integral, que se encuentra en el tercer y cuarto año de carrera, comprende las actividades destinadas a resolver los problemas de mayor complejidad y la rehabilitación de los problemas de salud en infantes y adultos, que han sido diagnosticados y referidos desde los niveles de atención anteriores. Se utilizan los procedimientos estomatológicos más adecuados en cada caso, extendiéndose a las fases quirúrgicas, estéticas y de rehabilitación, bajo la supervisión de un equipo de docentes. Se mantiene la estructura de

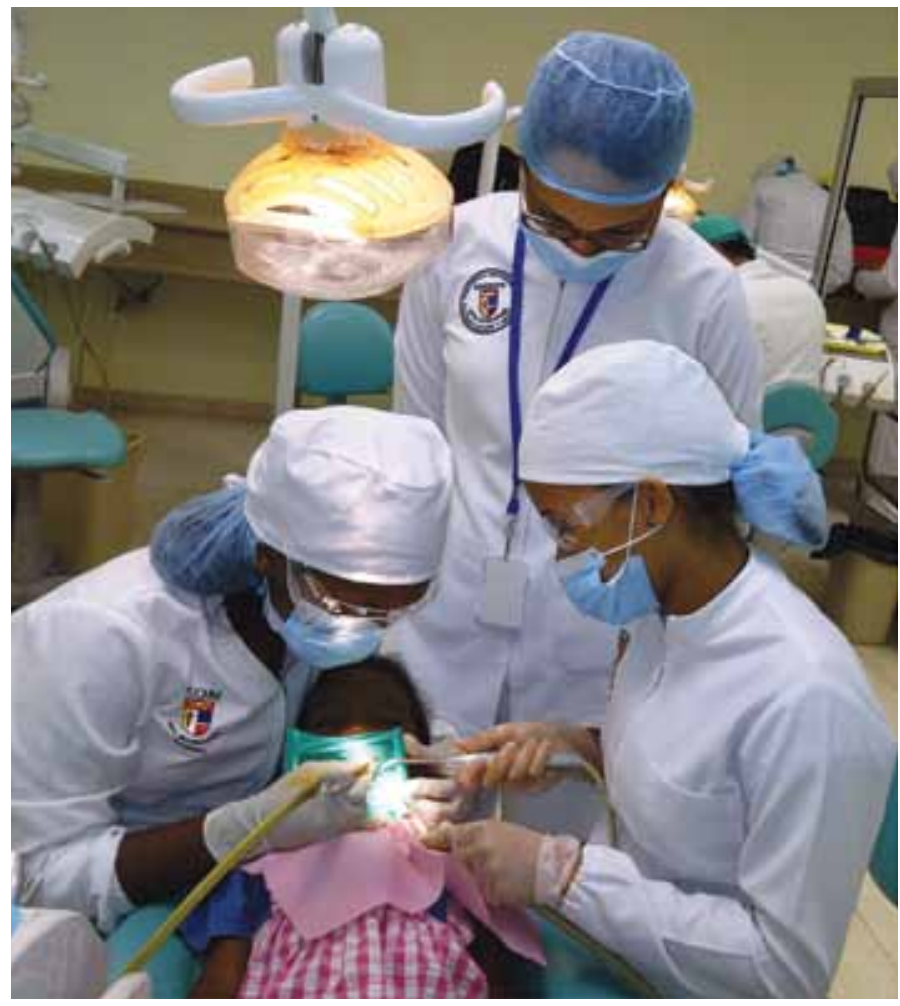

La enseñanza-aprendizaje en las prácticas de Estomatología, Karina Gutiérrez

Cuaderno de Pedagogía Universitaria Año 8/ N. 15 / enero - junio 2011 / Santiago, Rep. Dom.: PUCMM / pp.33-35 
supervisión de un docente encargado y un grupo de especialista de las principales áreas. En las prácticas de los tres niveles, las intervenciones docentes son puntuales, salvo en aquellos casos de determinada complejidad que lo ameriten. Por lo general, los docentes solo observan, orientan y dan pautas de manera instructiva, no operativa.

Una vez se inicia el nivel de Atención Primaria, se crea un expediente para cada estudiante, cada semestre se llena el formulario correspondiente al estudiante, quedando plasmada en el mismo la cantidad de actividades que se realizaron, los aspectos positivos, aspectos a mejorar y la calificación. De acuerdo a la complejidad del módulo, el estudiante debe alcanzar una determinada cantidad de prácticas que se evalúan de acuerdo a la calidad con que se realizan las mismas. Cada grupo de actividades tiene un valor asignado correspondiente a la calificación final. Los docentes van orientando a cada estudiante, puntualizando en el trascurso o después de cada actividad los puntos a mejorar, si es necesario; además de realizar dos evaluaciones controles en el trascurso para ir ayudando al estudiante. Se hace al estudiante responsable de su proceso, pues son ellos quienes deben indagar hasta obtener el diagnóstico y ofrecer al paciente el tratamiento correcto. Con este proceso se evidencia que el estudiante conoce su condición de desempeño y se esfuerza por ir mejorando día a día.

Cada docente lleva un diario de evaluación de las prácticas clínicas. De forma cualitativa y cuantitativa (en este último caso asignando un valor de acuerdo a una escala previamente elaborada) se deja plasmado el resultado de cada actividad que el estudiante realiza. Una vez el estudiante termina la actividad, el docente realiza los co- mentarios al mismo, aspectos a mejorar, habilidades desarrolladas de manera correcta, calidad y tiempo de trabajo. En algunas actividades es de gran importancia la valoración de los conceptos teóricos previos a la realización de dicha actividad, por la complejidad que estas ameritan, ya sea con la entrega de un reporte escrito o con la una evaluación oral. De igual manera, son evaluados los objetivos afectivos, que comprenden el proceso de atención al paciente, la relación colaborativa con la pareja asignada y con el grupo, con el docente y con el personal administrativo que se encuentra en la clínica.

Por tanto, es a través del proceso de integración teoría-práctica y del seguimiento y la evaluación constante que la carrera de Estomatología de la PUCMM pretende formar profesionales responsables y con las competencias necesarias para trabajar con principios éticos y con calidad disciplinar.

\section{Referencias bibliográficas}

Salazar, J. (2007). Desarrollo psicomotor en prótesis fijas. Acta odontológica venezolana 45, 3. Extraído 9 de mayo de 2011, de http:// www.scielo.org.ve/scielo.php?pid=S000163652007000300007 \&script=sci_arttext

Suárez, L. (2004). La enseñanza de la salud pública en la carrera de estomatología en Cuba. Revista Cubana de Salud Pública 30, 2. Extraído el 8 de mayo de 2011, de http://bvs.sld.cu/revistas/spu/vol30_2_04/ spu11204.htm 